\title{
RetComm 1.0: Real Time Condition Monitoring of Rotating Machinery Failure
}

\author{
Lee Chun Hong ${ }^{1}$, Abd Kadir Mahamad ${ }^{1,2, *}$, and Sharifah Saon ${ }^{1,2}$ \\ ${ }^{1}$ Faculty of Electrical and Electronic Engineering, Universiti Tun Hussein Onn Malaysia \\ ${ }^{2}$ Internet of Things Focus Group, Faculty of Electrical and Electronic Engineering, Universiti Tun Hussein Onn Malaysia
}

\begin{abstract}
The breakdown of motor proves to be very expensive as it increases downtime on the machines. Development of cost-effective and reliable condition monitoring system for the protection of motors to avoid unexpected breakdowns is necessary. Therefore, RetComm 1.0 is developed as assistant tool for bearing condition diagnosis system. The smartphone accelerometer is used to collect the vibration signal data and send it to computer by using the Android application named Matlab Mobile. The Matlab software is used to implement a program which is the RetComm 1.0 system to analyse the vibration signal and monitor the condition of the bearing. The algorithm used to observe the condition of bearing is trained by using Artificial Neural Network (ANN). In this project, the ANN is trained by using Matlab software. This proposed method is implemented for early diagnosis purposes. The diagnosis process can be done by just attached the smartphone onto the bearing for data collection. In conclusion, the bearing condition can be identified with this system. The bearing condition are shown in text to let the user know the bearing conditions. The raw data and power spectrum graph plotting are to let the user more further to understand the health condition of the bearing.
\end{abstract}

\section{Introduction}

The breakdown of motor proves to be very expensive as it increases downtime on the machines. So it becomes necessary to develop some cost-effective and reliable condition monitoring system for the protection of motors to avoid unexpected breakdowns [1]. Condition monitoring techniques which available in the market are obvious choice for the industries. Early prediction provides engineers information scheduling the maintenance [2].

Based on the previous researchers, the condition monitoring methods of the bearing faults are included temperature, acoustic emission, grindings, vibration and oil film resistance [3-6]. Vibration diagnosis is the most effective method and widely used at this present time.

The rolling element bearing is one of the most important components in the rotating machinery which contain of inner race, outer race, ball and cage. Figure 1 shows the basic structure of the rolling bearing.

The defect location of bearing can be determined by calculating the defect frequencies using these 4 equations [7].

$$
\begin{aligned}
& B P F I=\frac{N}{2} F\left(1+\frac{B}{P} \cos \theta\right) \\
& B P F O=\frac{N}{2} F\left(1-\frac{B}{P} \cos \theta\right)
\end{aligned}
$$

$$
\begin{gathered}
F T F=\frac{F}{2}\left(1-\frac{B}{P} \cos \theta\right) \\
B S F=\frac{P}{2 B} F\left(1-\left(\frac{B}{P} \cos \theta\right)^{2}\right)
\end{gathered}
$$

where BPFI, BPFO, FTF and BSF are the inner race defect frequency $(\mathrm{Hz})$, the outer race defect frequency $(\mathrm{Hz})$, the cage defect frequency $(\mathrm{Hz})$, and the ball spin frequency $(\mathrm{Hz})$, respectively. While, $N$ is number of balls bearing, $F$ is shaft frequency $(\mathrm{Hz}), B$ is ball diameter $(\mathrm{mm}), P$ is pitch diameter $(\mathrm{mm})$ and $\theta$ is contact angle.

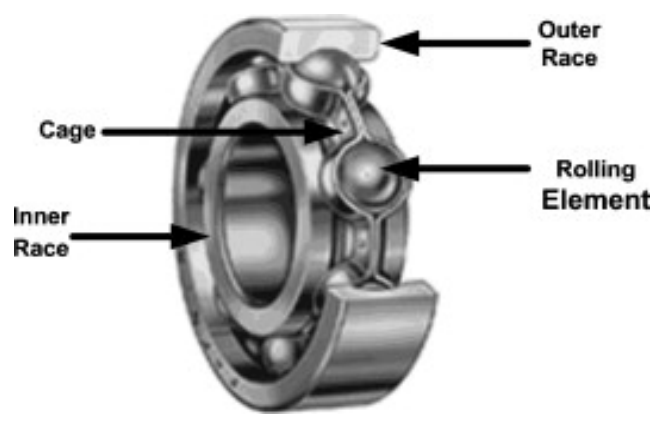

Fig. 1. The basic structure of rolling bearing

\footnotetext{
*Corresponding author: kadir@uthm.edu.my
} 


\section{Methodology}

Figure 2 illustrates the block diagram of the overall system and the figure 3 shows the flowchart of system architecture for RetComm 1.0 system. Initially, the system will measured the vibration signal of the machine by using the accelerometer application named Matlab Mobile and the raw data is save in .mat format file. Sugru is a self-setting rubber and almost hold everything. The Sugru is used to hold the smartphone on the machine instead of other holding items because it not absorb the vibration and affect the result. It stickiness is strong enough and easy to take off. The raw data is send to PC from smartphone via Wi-Fi Direct.

Matlab software is used as the back-end of the system and also to analyse vibration signal from the accelerometer. An algorithm used to calculate the condition of bearing is trained by using Artificial Neural Network. A Graphic User Interface (GUI) standalone program is implemented and used as the front-end system for users to review.

\subsection{The implementation of backend system}

The implementation of back-end system can be divided into 2 phases, the data collection phase and data analysis phase.

For data collection phase, the Matlab Mobile is installed to the smartphone for the measuring of the vibration signal. Matlab Mobile is an all-in-one diagnostic tools that record the status of the sensors inside the smartphone and accelerometer is one of it. The Matlab Mobile can direct send the accelerometer data into computer and save the data in Matlab format. Figure 4 shows the Matlab Mobile that collecting the status of the accelerometer inside the smartphone.

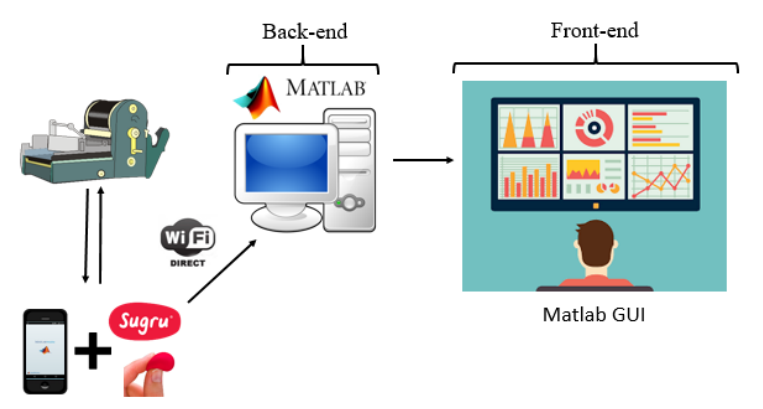

Fig. 2. Block diagram of the system

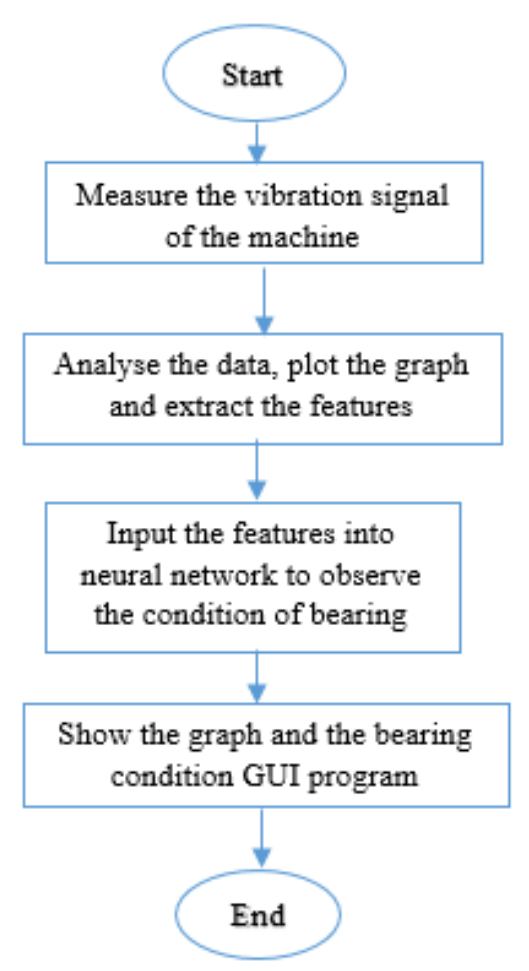

Fig. 3. The flowchart of the system

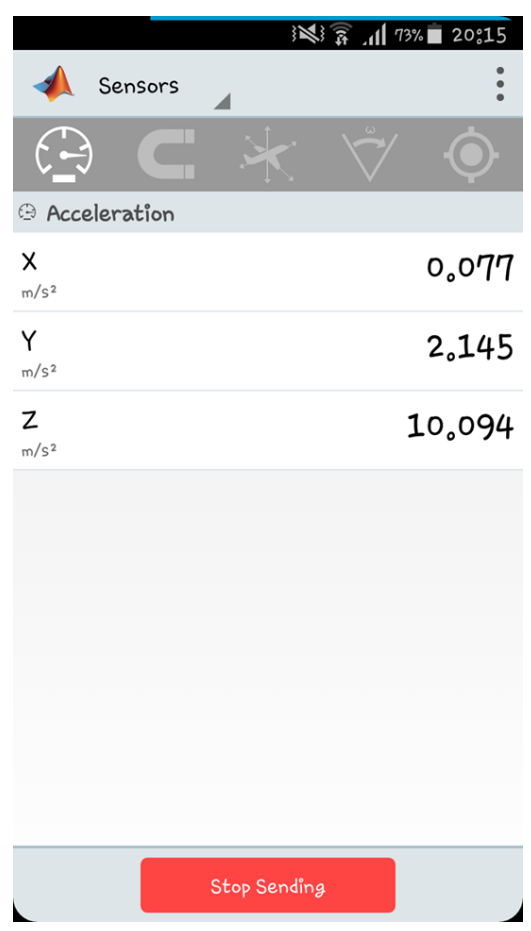

Fig. 4. The Matlab Mobile Apps that measuring the vibration signal 
To enable the connection between the Matlab software from computer and Matlab Mobile Apps from smartphone, the Matlab software in computer must have the support packages for Android Sensor.

For data analysis phase, the analysis part is divided into 2 parts, the vibration signal analysis and the bearing condition analysis. After applied vibration signal analysis, the raw data and power spectrum graph of the vibration signal are plotted and the results are generated. The generated results are the eight characteristics of the vibration signal which will be used in bearing condition analysis. These nine characteristics of the vibration signal are:
a) Maximum value
b) Minimum value
c) Kurtosis
d) RMS
e) Median
f) Variance
g) Standard deviation
h) Maximum envelope frequency
i) Maximum power spectrum

In previous studies that have been done by other researchers, the ANN is used to monitor the bearing condition and detect the bearing faults [8-11]. The inputs for the ANN are the extraction features from the data collected. By collecting the features from different conditions of the bearing, the neural network is trained with these features. Therefore, the evaluation result by the ANN is very accurate and trustable. In this project, neural network is used to train an algorithm for bearing condition analysis by input the eight features of the vibration signals.

The condition of bearing that can be monitored by Retcomn 1.0 are divided into four conditions, which are normal condition as class 1 , ball defect as class 2 , inner race defect as class 3 and outer race defect as class 4 . After finished the ANN network training, the best network will be selected as an optimum network, to generate it as standalone Matlab function for the implementation of GUI program.

\subsection{The implementation of frontend system}

The main role of the front-end of this system is to visualize the data and give to the end user for review. The standalone GUI program is used to visualize the data and act as the front-end for this system. The graphs which visualized are the raw data and the frequency spectrum of the vibration signal. The figure 5 shows the overview of the front-end system.

There are two push buttons in this GUI program which are the analysis button and clear button. The analysis button is to analyse the collected vibration signal data and be used to plot raw data and power spectrum graphs. The raw data graph is plotted into axes1 box meanwhile, the power spectrum graph is plotted into axes2 box. Besides, it also extract the features of the vibration signal data and put into neural_function. The function is loaded the features and check the bearing condition. The condition of the bearing is showed out in the static test box. The neural_function which is trained from the ANN network training is coded under analysis button. The codes for data analysis is also inside analysis button. The clear button is to reset the GUI layout into default layout and ready for next analysis.

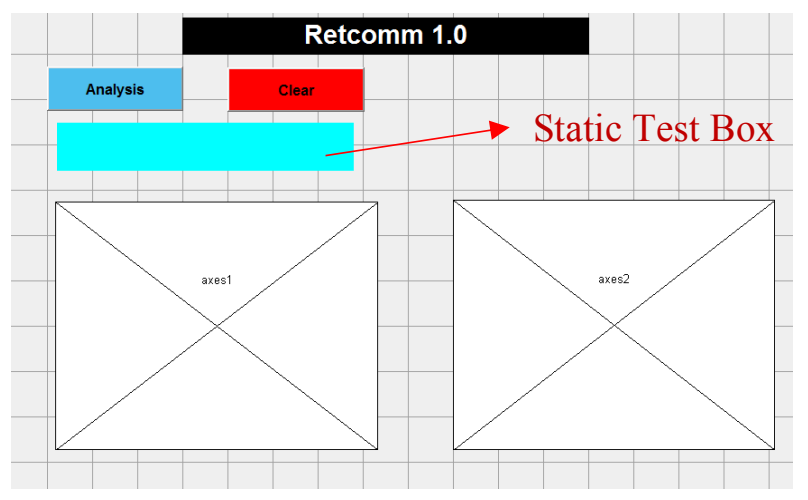

Fig. 5. The overview of the RetComm 1.0 GUI program

\section{Results}

\subsection{Accelerometer data analysis}

Figure 6 shows the plotting graph of raw data in Matlab. While, figure 7 shows the plotting graph of power spectrum in Matlab. The vibration signal used is the example of the normal condition of bearing.

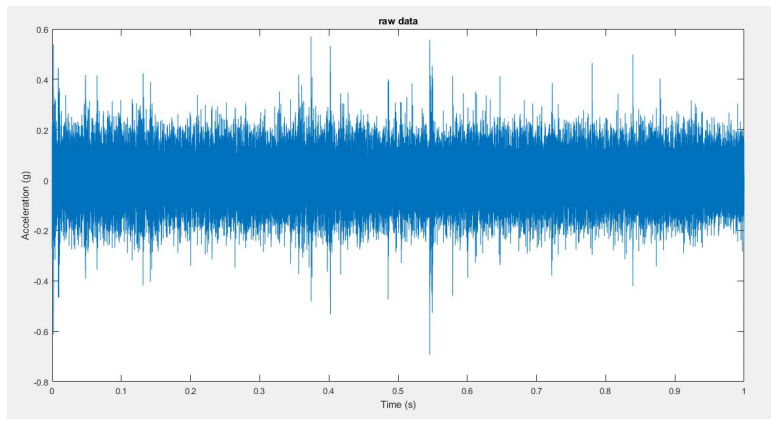

Fig. 6. The raw data graph

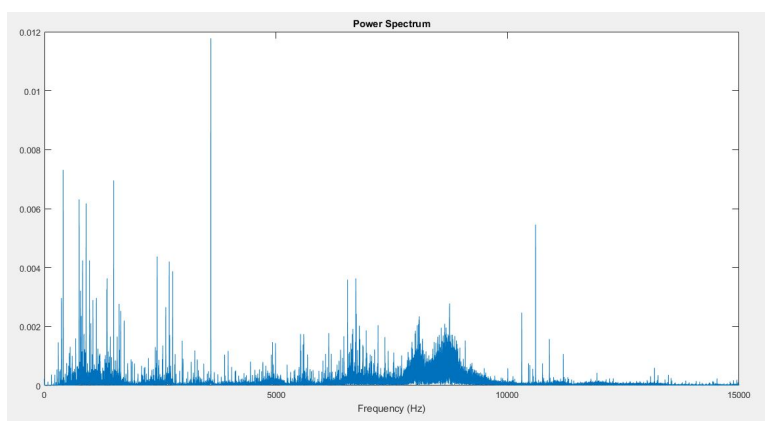

Fig. 7. The power spectrum graph 


\subsection{ANN network training}

Figure 8 shows the combination plot of the ANN network training results. The dashed line represents the perfect result, which are outputs $=$ targets. The solid line represents the best fit linear regression line between outputs and targets. The R-value is an indication of the relationship between the outputs and targets. From the figure 8 , the $\mathrm{R}$ is equals to 1 , which indicates that there is an exact linear relationship between outputs and targets.

\subsection{RetComm 1.0 frontend system}

Figure 9 shows the RetComm 1.0 GUI program layout. The raw data graph and power spectrum graph are plotted out and the bearing condition are shown up in static test box after pressed the analysis button. The clear button also function very well.

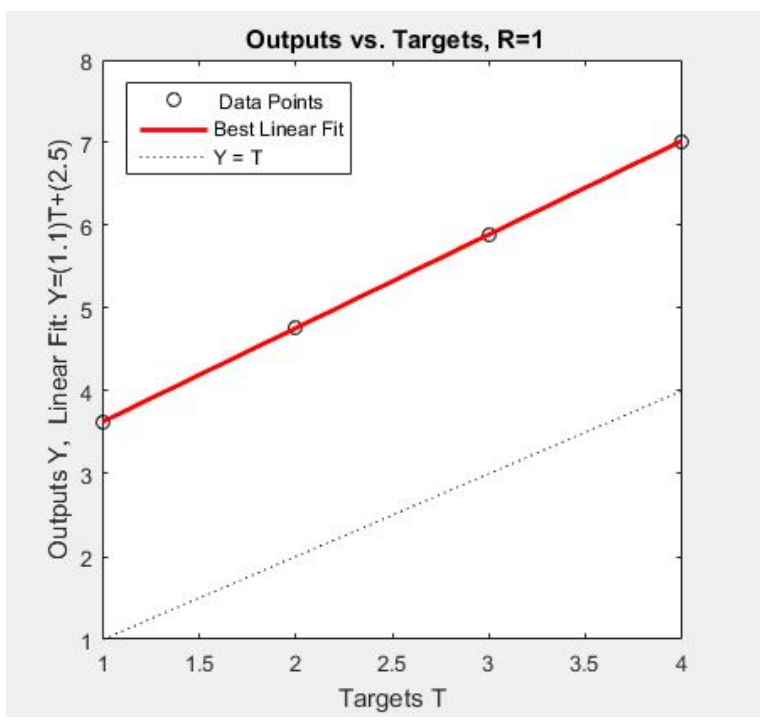

Fig. 8. The combination plot of the ANN network training results

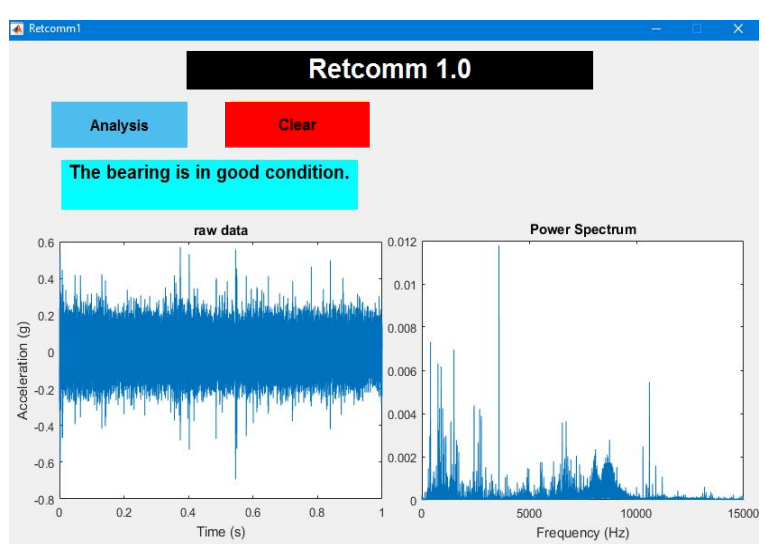

Fig. 9. The RetComm 1.0 GUI program layout

\section{Discussion}

For the raw data graph, the sampling frequency is 8000 samples/second and the length of time data sampled is 1 second. It mean the raw data is sampled 1 -second frame with 8000 samples. The values of raw data are the acceleration of the motor vibration. The power spectrum is calculated by applying Fast Fourier Transform (FFT) into the vibration signal data.

The neural network is trained with nine features of the vibration signal as input and give the result of the class as the bearing condition. The hidden layer of the network used tansig (tangent-sigmoid) transfer function and the output layer used purelin (linear) transfer function. This is to configure the network can approximate to any function. For the ANN network training technique for optimization, the Levenberg Marquardt (trainlm) algorithm is used because it gives the better performance among the other network training algorithms. By train it with different number of hidden nodes from 2 to 20 , the least validation error among them implies that it is the best neural network, which are with 4 hidden nodes and $2.10 \mathrm{E}-2$ validation error, as shown in table 1.

Table 1. The number of hidden nodes trained with respective of training error, validation error and testing error

\begin{tabular}{|c|c|c|c|}
\hline No. of Nodes & Training & Validation & Testing \\
\hline 2 & $2.15 \mathrm{E}-01$ & $2.57 \mathrm{E}-01$ & $3.55 \mathrm{E}-01$ \\
\hline 3 & $7.89 \mathrm{E}-02$ & $1.19 \mathrm{E}-01$ & $2.73 \mathrm{E}-01$ \\
\hline 4 & $3.75 \mathrm{E}-10$ & $2.10 \mathrm{E}-02$ & $8.50 \mathrm{E}-02$ \\
\hline 5 & $4.80 \mathrm{E}-03$ & $1.02 \mathrm{E}-01$ & $3.98 \mathrm{E}-01$ \\
\hline 6 & $1.75 \mathrm{E}-04$ & $2.30 \mathrm{E}-02$ & $9.87 \mathrm{E}-02$ \\
\hline 7 & $3.41 \mathrm{E}-04$ & $2.35 \mathrm{E}-01$ & $1.25 \mathrm{E}+00$ \\
\hline$\vdots$ & $\vdots$ & $\vdots$ & $\vdots$ \\
\hline 19 & $4.29 \mathrm{E}-02$ & $9.94 \mathrm{E}-02$ & $4.61 \mathrm{E}-01$ \\
\hline 20 & $2.05 \mathrm{E}-02$ & $8.51 \mathrm{E}-02$ & $2.86 \mathrm{E}-01$ \\
\hline
\end{tabular}

\section{Conclusion}

Through the completion of RetComm 1.0 system, the objective of this project which is to design RetComm 1.0 system that helps to monitor the condition of bearing has been achieved. In addition, the objective which is to analyze the performance of this system has been achieved as well in this project.

With RetComm 1.0 system, the user can monitor the condition of the bearing by just attach the smartphone onto the machine for a few minutes. It can help to save a lot of time to measure all of the machine in a certain area in the industry which are in a huge number of rotating machinery. By using accelerometer, the affected machine is no need to be shut down in order to carry out the diagnosis process. In this case for further investigation, industries required expert supervisor/technician to predict the condition of that 
bearing through vibration signal or power spectrum analysis and other diagnostic tools such as acoustic emission and tomography. It may cause the company lost in terms of profit and time. By using RetComm 1.0, it can diagnosis the machine in simple way without spent a lots of time for investigation and not required an expert to decide the machine condition.

For the analysis of this system performance, the bearing condition can be identified after insert the features of the vibration data of the machines. The bearing condition are shown in text to let the user know. The raw data and power spectrum graph plotting are to let the user more further to understand the health condition of the bearing. To use this system, the user just need the install the software application into the computer.

The financial support received from Office for Research, Innovation, Commercialization and Consultancy Management (ORICC), Universiti Tun Hussein Onn Malaysia is gratefully acknowledged.

\section{References}

1. M. Irfan, N. Saad, R. Ibrahim, V.S. Asirvadam, An intelligent diagnostic system for the condition monitoring of AC motors, 8th IEEE Conference on Industrial Electronics and Applications (ICIEA), pp. 1248-1253 (2013)

2. H. Wasif, A. Aboutalebi, D. Brown, L. Axel-Berg, Condition monitoring system for process industries a business approach, IEEE Symposium on Industrial Electronics and Applications (ISIEA), pp. 251-256 (2012)

3. M.A.A. Elmaleeh, N. Saad. Acoustic emission techniques for early detection of bearing faults using LabVIEW, 5th International Symposium on Mechatronics and Its Applications, pp. 1-5 (2008)

4. Ling Xiang, Lanlan Hou, Feature analysis of interaction on rub-impact and oil-film faults for a rotor-bearing system, 12th International Conference on Ubiquitous Robots and Ambient Intelligence (URAI), pp. 246-254 (2015)

5. G.A. Skrimpas et al. Detection of generator bearing inner race creep by means of vibration and temperature analysis, IEEE 10th International Symposium on Diagnostics for Electrical Machines, Power Electronics and Drives (SDEMPED), pp. 303-309 (2015)

6. Wei Feng et al., Modeling and Simulation of Process-Machine Interaction in Grinding of Cemented Carbide Indexable Inserts, Hindawi Publishing Corporation, Shock and Vibration, Article ID 508181 (2014)

7. B.P. Graney, K. Starry, Rolling Element Bearing Analysis, The American Society for Nondestructive Testing Inc., Materials Evaluation, 70(1), pp. 78-85 (2012)
8. L.S. Dhamande, M.B. Chaudhari, Detection of Combined Gear-Bearing Fault in Single Stage Spur Gear Box Using Artificial Neural Network, Procedia Engineering, Elsevier, 144, pp. 759-766 (2016)

9. Z.X. Li, J.J. Zhu, X.F. Shen, C. Zhang, J.W. Guo, Fault Diagnosis of Motor Bearing Based on the Bayesian Network, Procedia Engineering, Elsevier. 16, pp. 18-26 (2011)

10. H.S. Kumar, P.P. Srinivasa, N.S. Sriram, G.S. Vijay, ANN-based evaluation of performance of wavelet transform for condition monitoring of rolling element bearing, Procedia Engineering, Elsevier, 64, pp. 805- 814 (2013)

11. A.K Mahamad, T. Hiyama, Diagnosis and Prognosis of Bearing Failure in Rotating Machinery using Acoustic Emission and Artificial Neural Network, IEEJ Transactions on Industry Applications, 130, pp. 443-449 (2009) 